\title{
NEAMS Software V\&V Plan for the MARMOT Software
}

\author{
Michael R. Tonks
}

March 2014

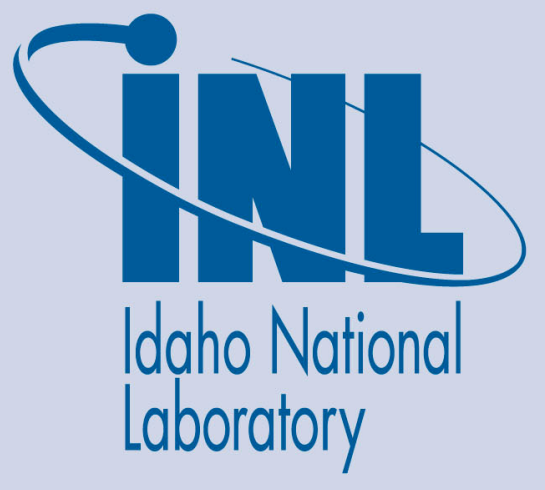

The INL is a U.S. Department of Energy National Laboratory operated by Battelle Energy Alliance 
INL/EXT-14-31695

\title{
NEAMS Software V\&V Plan for the MARMOT Software
}

\author{
Michael R. Tonks
}

March 2014

\section{Idaho National Laboratory \\ Idaho Falls, Idaho 83415}

http://www.inl.gov

\author{
Prepared for the \\ U.S. Department of Energy \\ Office of Nuclear Energy \\ Under DOE Idaho Operations Office \\ Contract DE-AC07-05ID14517
}




\section{NEAMS SOFTWARE V\&V PLAN FOR THE MARMOT SOFTWARE}

\section{Introduction}

The US Nuclear Energy Advanced Modeling and Simulation (NEAMS) Program is investing in the development of a next generation fuel performance modeling capability as part of its Fuels Product Line. This new capability is provided by the MOOSE-BISON-MARMOT (MBM) suite of codes. MOOSE (Multiphysics ObjectOriented Simulation Environment) is a finite element based framework that provides advanced numerical tools for the rapid development of advanced simulation applications. BISON, the engineering scale fuel performance tool, is based on the MOOSE framework and predicts the performance of various reactor fuels types in 1D, 2D, and 3D. Finally, MARMOT is a mesoscale multiphysics tool that predicts the coevolution of microstructure and properties within reactor fuel throughout its lifetime in the reactor. Together, these tools facilitate the predictive modeling of nuclear fuel performance to assist in the design and analysis of reactor systems and components.

Though BISON was initially stood up using legacy materials models that are primarily empirical fits to experimental data, these models cannot provide the predictive capability required in a next generation fuel performance code. Empirical models cannot accurately extrapolate out of their test bounds, and therefore are not applicable to accident conditions or new reactor fuel concepts. In addition, these empirical models are typically correlated to burnup to quantify the history of the material in the reactor. However, burnup is not a unique measure of the material history, as fuel can reach the same burnup from different conditions, and thus have different microstructures and properties. To overcome these limitations, multiscale modeling and simulation are being used to supplement difficult to obtain experimental data to develop materials models based on microstructure rather than burnup. Atomistic simulations are used to determine mechanisms and properties, which are then incorporated into MARMOT to model the radiation-induced microstructure evolution and its impact on the fuel material properties.

In order to ensure the accuracy and quality of the microstructure based materials models being developed in conjunction with MARMOT simulations, MARMOT must undergo exhaustive verification and validation. Only after this process can we confidently rely on the MARMOT code to predict the microstructure evolution within the fuel. Therefore, in this report we lay out a V\&V plan for the MARMOT code, highlighting where existing data could be used and where new data is required. As MARMOT development is ongoing, this report will also evolve with time. We will update the report annually, as more MARMOT simulations are developed and as new data is collected. It is an open document and is freely 
available to interested researchers within the US.

\section{Acronyms}

NEAMS - Nuclear Energy Advanced Modeling and Simulation

INL - Idaho National Laboratory

MOOSE - Multiphysics Object Oriented Simulation Environment

MBM - MOOSE-BISON-MARMOT

$\mathrm{PF}$ - Phase field

GB - Grain boundary

MD - Molecular Dynamics

DFT - Density Functional Theory

$\mathrm{V} \& \mathrm{~V}$ - Verification and validation

NEUP - Nuclear Energy University Partnership

FCRD - Fuel Cycle Research and Development

ATR - Advanced Test Reactor

HFIR - High Flux Isotope Reactor

EDS - Energy dispersive spectroscopy

EBSD - Energy Backscatter Diffraction

SNL - Sandia National Laboratory 


\section{Table of Contents}

1 Introduction

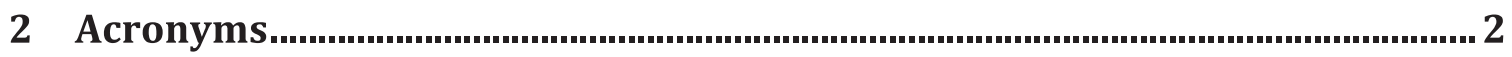

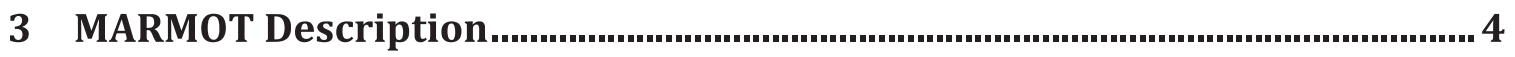

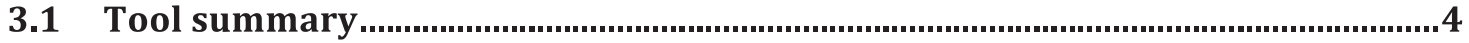

3.2 Consequence of failure …….........................................................................

3.3 Code dependencies: Software and Hardware .....................................................

4 Model Verification and Validation ......................................................................6

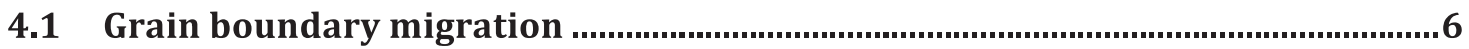

4.1.1 Verification ...............................................................................................................

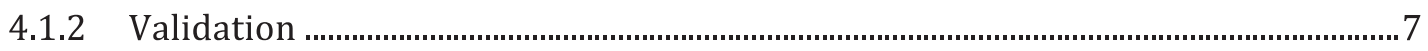

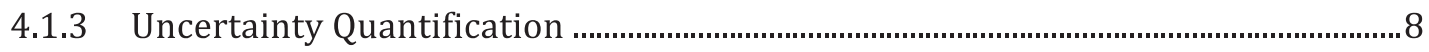

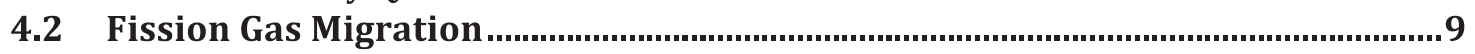

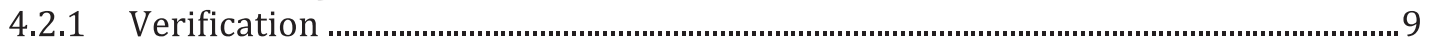

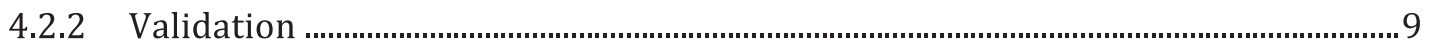

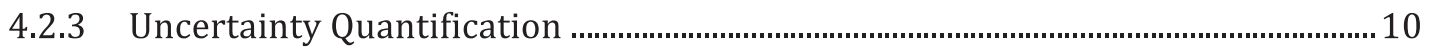

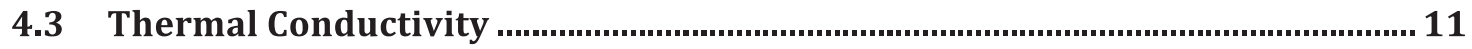

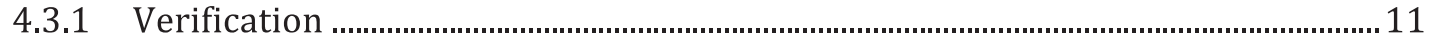

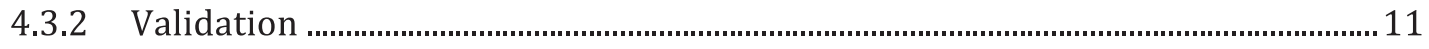

4.3.3 Uncertainty Quantification ..........................................................................................12

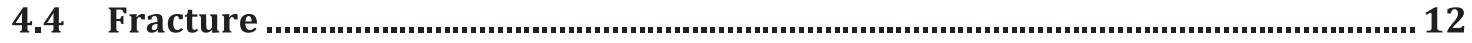

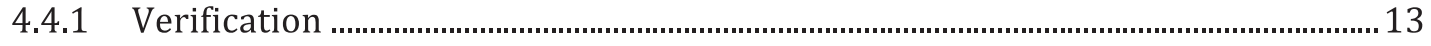

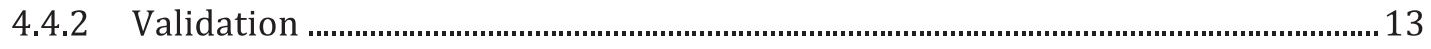

4.4.3 Uncertainty Quantification .......................................................................................14

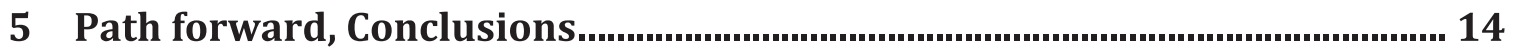

6 References and Additional Material................................................................. 16 


\section{MARMOT Description}

Here we describe the MARMOT code, summarizing the models used to predict microstructure evolution and its impact on fuel properties. We also discuss the impact of incorrect predictions by MARMOT and its potential impact on the BISON code.

\subsection{Tool summary}

MARMOT is a multiphysics mesoscale code focused on modeling irradiation-induced microstructure evolution in reactor materials [3.1.1]. It is based on the MOOSE framework and uses the basic phase field classes that are built into the phase field module in MOOSE (MOOSE-PF) [3.1.2]. The phase field method is used to model the microstructure evolution and it is coupled to finite deformation mechanics and heat conduction to capture the multiphysics nature of the microstructure evolution. The impact of the evolution on the material properties is quantified using advanced homogenization approaches to take the local material properties at the mesoscale and determine a single effective property over the entire mesoscale domain.

The phase field method is used in MARMOT due to its flexibility in modeling many coupled categories of microstructure evolution. In the phase field model, microstructural features are represented by continuous variables, which are evolved to minimize a function describing the free energy of the system [3.1.3]. It has been used to model a large range of behaviors, including phase change, solidification, GB migration, and radiation damage. In MARMOT, the phase field model has been directly applied to model radiation damage in $\mathrm{UO}_{2}$ and zircaloy cladding. The phase field model is coupled to linear elasticity to model the impact of stress on the fuel behavior, and to finite strain mechanics to model the impact of deformation in zirconium alloys. The phase field models are also regularly coupled to heat conduction, where the local thermal conductivity varies locally across the microstructure.

When developing a phase field model, the probable dominant mechanisms must be built into the model. Therefore, it is critical that the dominant mechanisms be known, or the model will not accurately predict the material behavior. In addition, the phase field model requires material properties that can be difficult to measure experimentally. Thus, atomistic modeling plays a critical role in mesoscale modeling, both to determine the dominant mechanisms and to evaluate needed parameters [3.1.4].

The MARMOT simulations are then used to develop a mechanistic understanding of the behavior taking place at the mesoscale. This information is used to develop new materials models that account for the lower length scale information provided by MARMOT and the atomistic simulations. These mechanistic materials models are used in BISON to provide a modeling capability based on microstructure rather than burnup that will be truly predictive. 


\subsection{Consequence of failure}

MARMOT simulations are used to gain a basic understanding of the material behavior, supplementing difficulty to obtain experimental data. However, if predictions are incorrect or have significant error, the predicted behavior will lead to erroneous understanding of the materials behavior. These errors can result from three main sources, or any combination of the three:

1. An error was made in the implementation of the model

2. A critical mechanism was not included in the model.

3. The parameters values are incorrect.

As MARMOT becomes more established and is used in the design and interpretation of experiments, these errors could result in incorrect results and conclusions.

The largest impact of errors in MARMOT will be in the BISON materials models being developed using MARMOT results as a reference. As BISON is being developed as a production code that could be used in the design and certification of reactor fuel, errors in the materials models could result in incorrect predictions. However, BISON undergoes substantial validation and assessment, and therefore incorrect materials models will most likely be identified at the BISON level. Yet, these incorrect models caused by erroneous MARMOT calculations could cost significant time and resources.

\subsection{Code dependencies: Software and Hardware}

MARMOT is based on the MOOSE framework and has all of the same code dependencies as MOOSE. Thus, MARMOT requires the following libraries to run:

- MOOSE

- LibMesh

- Petsc

- Hypre

- MPI

All of these libraries must be downloaded and compiled in order to run MARMOT. To make this easier, the MOOSE team maintains a compiled module of all dependencies except MOOSE on the INL high performance computing system and precompiled packages containing Petsc, Hypre, and MPI for MacOS, Ubutu, and OpenSuse.

MARMOT, due to its dependence on MOOSE, is not compatible with Windows operating systems. However it is fully compatible with Linux, Unix, and MacOS. It is a flexible code that can be used for small problems that can be run on a desktop or laptop, as well as large problems that require a supercomputer. Typically, small 2D problems can be run on a laptop computer. Medium to large 2D problems can be run on a powerful desktop. 3D problems typically require a cluster with hundreds to thousands of processors.

In order to minimize the impact of operating system and code architecture on the performance of MARMOT, MARMOT employs the rigorous quality assurance 
structure developed for the NQA-1 certified MOOSE framework. For each capability within MARMOT, a test problem has been checked into the MARMOT repository, along with a "gold" result file that represents the optimal result. Currently, there are more than 100 tests for MARMOT. Any time any change is made to MARMOT or to MOOSE, all the tests are run on five different architectures, using two different operating systems and three different compilers. If any of the results from these tests do not match the "gold" file, an alert is issued and the problem is identified and repaired.

\section{Model Verification and Validation}

In this section, we summarize the four main physical phenomena that have been the focus of the MARMOT development funded under NEAMS. Other MARMOT development funded by other programs (e.g. zircaloy) will not be discussed in this document. For each phenomenon, verification and validation will be discussed. In this report, verification is defined as the process of ensuring that the code accurately solves the implemented mechanisms, while validation is defined as the process of ensuring that the model provides results consistent with experimental data. Each model must undergo both verification and validation. Verification is used to identify errors from source 1 (from section 3.2) while validation identifies errors due to sources 2 and 3.

\subsection{Grain boundary migration}

GBs migrate to reduce the overall free energy of the system. The driving forces for this migration can include reducing the GB energy, the elastic energy, the dislocation energy, or more. GBs are more mobile at high temperature, thus the average grain size of reactor fuel tends to grow in the hotter portions of the fuel. However, particles and pores within the material can pin GB migration, slowing or even halting GB migration. Grain growth within the fuel has a significant impact on fission gas release, swelling, and cracking.

GB migration and grain growth has been a large focus area for MARMOT since it was created first [3.1.1, 4.1.1, 4.1.2]. A typical phase field model for grain growth [4.1.3] has been implemented in MARMOT, and advanced numerical algorithms have been developed to increase the numerical efficiency of the model. Thus, thousands of grains can be modeled in 2D and 3D. Various driving forces for GB migration have been modeled in MARMOT, including GB energy (curvature driving force), elastic energy, and temperature gradient. Our primary material focus has been on $\mathrm{UO}_{2}$, though we have modeled grain growth in $\mathrm{Cu}$ and $\mathrm{Mo}$, as model materials with available parameters. All of our simulations have assumed isotropic GB properties, though a collaborator from the University of Wisconsin, Madison has implemented an anisotropic model.

A model of particle and pore pinning has also been implemented in MARMOT. This model defines the pinning as a function of the ratio of the GB and interfacial energies. The model can be run with static pores/particles, where they do not evolve during 
the simulation, or it can be coupled with an additional model that evolves the pore/particles as they interact with the GBs.

The primary material properties required for the grain growth model are the GB energy and the GB mobility. These parameters vary with GB type, though we have assumed isotropic properties to simplify the model. The elastic driving force model also requires the single crystal elasticity tensor (note that this calculation is inherently anisotropic), while the temperature gradient model requires a typical GB width, the entropy difference between the GB and the matrix, and the molar volume of the material.

\subsubsection{Verification}

A well-established analytical model exists defining GB migration due to the curvature driving force. The MARMOT grain growth model has been compared to this analytical model, verifying that it accurately represents curvature driven grain growth [4.1.1]. Results that have been verified against the analytical model have been checked into the testing system as "gold" files, ensuring that the code always maintains a good comparison with the analytical model. The model of the temperature gradient driving force has also been compared to an analytical model and the results were found to underpredict the GB migration by $\sim 10 \%$ [4.1.2]. The pinning model has been compared to various analytical models and has been shown to compare well. More detail is given on the analytical models in the MARMOT theory manual.

To further verify the grain growth model, it has also been compared to results from MD simulations. As MD explicitly models the interactions between atoms, it provides a less "biased" result than the phase field model, as it does not require the incorporation of specific mechanisms. Our simulations of curvature driven grain growth were found to compare well with the MD simulation results [4.1.1]. Comparison to MD has also been made for the temperature gradient driving force, and the results were consistent with the comparison to the analytical model. Finally, the pore/particle interaction model was compared with the MD simulation results. The comparison was good, except that the bubbles were dragged slightly by the GBs in the MD simulations, while this did not occur in the phase field simulations.

\subsubsection{Validation}

While there is uncertainty in the model and a great need for validation, no direct comparison to experimental data has been conducted. While there are various papers that have conducted grain growth experiments on $\mathrm{UO}_{2}$, they have always used sintered fuel pellets, and thus can only measure average values of the GB mobility and include pore pinning effects and impurity drag. However, to validate the separate GB driving force models in MARMOT will require more careful experiments that work to eliminate the impact of impurities, second phase particles, and pores.

In order to validate the $\mathrm{UO}_{2}$ grain growth models within MARMOT, we require new 
sets of experiments to be conducted. These experiments fall into two main categories: experiments using bicrystal samples (investigating the behavior of a single GB) and polycrystal samples with detailed 3D characterization. Both sets of experiments will investigate curvature driven GB migration, and the polycrystal experiments will investigate pore/particle pinning. The temperature gradient driving force appears to be negligible and therefore need not be investigated.

The main purpose of bicrystal experiments is to measure the properties of an individual GB. These bicrystal samples can be directly fabricated as bicrystals or be sectioned from large grain size samples using a focused ion beam. In situ experiments are possible, but due to the slow $\mathrm{GB}$ mobility of $\mathrm{UO}_{2}$ may be difficult to conduct. The temperature must be very high before the GB would move at a rate feasible to be observed in situ. To investigate the curvature driving force, a range of sample geometries have been identified that facilitate the quantification of the reduced GB mobility (the product of the GB energy and mobility) [4.1.4], and MARMOT has been used to assist in the design of these experiments [4.1.1]. An important result of these experiments is a relationship between GB type and GB mobility. MARMOT simulations could be directly compared to the results from these experiments to validate the curvature driving force model.

Two types of polycrystal experiments are needed. The first type are annealing experiments of polycrystalline $\mathrm{UO}_{2}$ with zero porosity, i.e. ideal or near ideal density. The initial texture and topology of the sample should be characterized in 3D using nondestructive techniques that provide information about crystal orientation. Then, at various intervals during the annealing, the sample could be removed and characterized again, given various snapshots of the grain growth. The second type are also annealing experiments, but with initial porosity ranging from $3 \%-10 \%$.

The polycrystalline $\mathrm{UO}_{2}$ samples without porosity would provide the perfect data to validate the curvature driven grain growth model on more than two grains. It would also provide the proper tool for evaluating the impact of isotropic GB properties on both the average and local grain growth behavior. The samples with porosity would be the proper avenue for validating the pore interaction model. The impact of assuming isotropic GB properties could also be evaluated for this model.

\subsubsection{Uncertainty Quantification}

The value for the GB mobility currently used to model $\mathrm{UO}_{2}$ grain growth was taken from an experimental paper [4.1.5]. However, these experiments were conducted on sintered $\mathrm{UO}_{2}$ pellets, and thus include impurity drag and porosity pinning in their measured value of the mobility. The GB energy value currently used is for a Sigma 5 GB and was determined using MD [4.1.6]. Thus, there is uncertainty in both of these values. There is even more uncertainty in the temperature gradient driving force and the pore/particle pinning models.

While additional experiments will help to reduce this uncertainty, it is still important to quantify how the parameter uncertainty propagates to uncertainty in 
the predicted grain behavior. No work has been published on quantifying the uncertainty of a phase field grain growth model, so this work would need to be conducted. DAKOTA, a tool for uncertainty quantification under development at Sandia National Laboratory, has been successfully used with BISON with excellent results. Thus, it should be fairly straight forward to apply DAKOTA to the grain growth model within MARMOT.

\subsection{Fission Gas Migration}

As atoms within the fuel fission to release heat, various fission products are produced. While some of these fission products form solid phases, others are gaseous and can transport through the material. They tend to segregate to voids to form bubbles. Small bubbles form within the grains and much larger bubbles form on the GBs, as the fission gas tends to segregate to GBs. The fission gas bubbles interconnect until the gas is able to leave the GB and enter a network of interconnect tunnels along triple junctions. Eventually, the gas reaches a free surface and is released. Fission gas has a large impact on the fuel thermal conductivity, swelling, fracture, and plenum pressurization.

Due to its importance, it is critical to model fission gas migration and segregation within the fuel. Atomistic simulations have been employed to investigate the transport of fission gas through the $\mathrm{UO}_{2}$ matrix and its segregation energy at GBs. MARMOT uses these values to quantify the impact of fission gas on the fuel performance. Phase field simulations have modeled the interactions between vacancies, interstitials, and fission gas [4.2.1]. MARMOT simulations have modeled fission gas segregation to GBs and intragranular bubble nucleation and growth during post-irradiation annealing [4.2.2]. On going work in MARMOT will model fission gas behavior in 3D polycrystals with a more mechanistic model of the fission gas behavior and the bubble pressure.

The primary material properties required to model fission gas behavior are the energetics of the formation and migration of fission gas atoms, vacancies, and interstitials. In addition, the diffusivity prefactors and the surface energy for fission gas bubbles are needed.

\subsubsection{Verification}

Analytical models exist to predict behavior related to fission gas. The fission gas transport at low concentrations can be verified by comparing to the solution of the diffusion equation. For the growth of a fission gas bubble, there exists an analytical $1 \mathrm{D}$ growth model that predicts the rate of growth given the bulk gas concentration and the diffusivity. Rate theory equations can also be used to verify the code and ensure that it is predicting the correct behavior. As with the grain growth model, verified results have been used as the "gold" standard in the testing system. More detail on these models is provided in the MARMOT theory manual.

\subsubsection{Validation}

Though fission gas behavior is a critical part of fuel performance, there is very little 
data at the microstructure level quantifying it due to the difficulty in characterizing irradiated materials. However, there have been some papers that have looked at both intra- and intergranular bubble behavior in irradiated materials. In fact, the basic fission gas bubble model has been validated by comparing to data from postirradiation annealing samples [4.2.2]. There are also data on GB bubbles that has been used to verify a phase field GB bubble model that was developed using NEAMS funding [4.2.3] that has now been implemented in MARMOT.

To fully validate the fission gas models within MARMOT requires separate effects data, where fission gas behavior is investigated in unirradiated materials. For such experiments, the fission gas must be added artificially to the material, as no actual fissioning is taking place. This has been accomplished by growing thick films in a Xe rich environment [4.2.4] or could be accomplished via ion implantation. Phenomena that need to be investigated using such an approach include:

- Diffusivity of Xe, $\mathrm{Kr}$, etc. in single (or large) crystal $\mathrm{UO}_{2}$

- Xe, Kr, etc. diffusion in bicrystal samples, to identify GB segregation energy.

- Bubble nucleation and early stages of growth.

- Diffusion of Xe, Kr, etc., in a temperature gradient, as well as bubble migration in a temperature gradient.

In addition to unirradiated separate effects experiments, Xe-ion irradiation experiments could be very important. Such experiments would investigate the same phenomena mentioned in the previous paragraph, but could investigate the impact of point defects on the diffusion and segregation by comparing to the unirradiated samples.

Finally, tightly controlled neutron irradiation experiments could provide critical insights. The microstructure of the samples would be characterized initially using EBSD, EDS, and other techniques in a section of the sample marked for later identification (i.e. with fiducial marks). Then, these samples would undergo short term irradiation, e.g. using a Rabbit system in ATR or HFIR. After the irradiation, the microstructure would again be characterized and compared to the initial microstructure to determine changes that have taken place. Such experiments would provide information on the migration and segregation of fission products and would be the final validation of the fission gas models within MARMOT.

\subsubsection{Uncertainty Quantification}

Due to the difficulty of characterizing the behavior of irradiated samples, there is a large amount of uncertainty in all of the parameters defining the fission gas behavior. We are using first principles calculations to determine some of the fission gas behavior to reduce some of the uncertainty, but there is still uncertainty in those calculations. Thus, it is critical to quantify the resultant uncertainty in all the fission gas calculations. Unfortunately, no uncertainty quantification on these models has been conducted to date. As with the grain growth, initial uncertainty quantification could be conducted using SNL's DAKOTA code. 


\subsection{Thermal Conductivity}

As the goal of a nuclear reactor is to convert heat produced from fission into energy, conducting the heat from the $\mathrm{UO}_{2}$ fuel to the coolant is a critical part of the fuel performance. In addition, thermal conductivity degradation causes the fuel centerline temperature to rise and can eventually lead to fuel melting. As the fuel is irradiated, its thermal conductivity decreases due to the generation of defects, fission products, and the formation of fission gas bubbles.

Various empirical and semi-empirical models have been developed to predict how the thermal conductivity evolves under irradiation. However, in an effort to create a microstructure based model of the fuel thermal conductivity, we have conducted MARMOT simulations in which we determined the effective thermal conductivity of a specific microstructure by locally varying the thermal conductivity to correspond to a specific microstructural feature [4.3.1]. For example, the thermal conductivity in the bulk $\mathrm{UO}_{2}$ is higher than across a GB and both are higher than in a fission gas bubble. The thermal conductivity is then homogenized to determine the effective thermal conductivity of the microstructure. This approach has been used to quantify the impact of fission gas bubbles along GBs on the thermal conductivity of $\mathrm{UO}_{2}$ [4.3.2, 4.3.3].

In order to accurately represent the local thermal conductivity of a $\mathrm{UO}_{2}$ microstructure, the single crystal thermal conductivity must be known. It is also necessary to know how point defects, off-stoichiometry, and dispersed fission gases reduce the bulk thermal conductivity. In addition, the GB thermal resistance, or Kapitza resistance, must be known for various GB types. Finally, the thermal conductivity across other microstructure features is needed, such as a fission gas bubble or solid fission products.

\subsubsection{Verification}

While there are some analytical mixture models to determine the effective thermal conductivity of a microstructure with other features, they are only applicable for certain shapes and configurations. For example, the Maxwell-Euken equation can be used to verify the thermal conductivity model for spherical particles or pores randomly distributed through the matrix. In addition, the Kapitza resistance model can be used to verify the predicted effective thermal conductivity of a bicrystal or polycrystal.

Basic verification of GB thermal resistance has been conducted in MARMOT. However, verification of the impact of bubbles on the thermal conductivity still needs to be conducted using various bubbles shapes and configurations.

\subsubsection{Validation}

The primary experimental research that has been conducted to evaluate the $\mathrm{UO}_{2}$ thermal conductivity has measured thermal conductivity across unirradiated bulk samples and irradiated samples as a function of burnup. These data do not quantify the microstructure in any way, therefore they are not suitable for validation for our 
MARMOT simulations. Therefore, new data is required to meet the validation needs.

The experiments required for MARMOT validation will cover a large range of samples with different microstructure features, but in each case we need thermal conductivity data from room temperature to $\sim 2500 \mathrm{~K}$. First, we need accurate measurements of the single crystal thermal conductivity in $\mathrm{UO}_{2}$ for stoichiometric and off-stoichiometric samples. Measurements are needed for various levels of hyper- and hypo-stoichiometry. Using bicrystal or large grain samples, measurements of the GB thermal resistance are needed for various GB types. In addition, data is needed on the impact of defects (point defects and defect clusters) on thermal conductivity. The thermal conductivity as a function of dispersed fission gas concentration also needs to be measured.

In order to compare the MARMOT capability to determine the effective thermal conductivity, thermal conductivity measurements are needed at the mesoscale. Such measurements should measure the thermal conductivity across a well-characterized microstructure, including porosity, grain structure, and chemical composition. Nondestructive measurement approaches could be used to characterize the microstructure before the thermal conductivity measurement, or destructive 3D approaches could be used after the thermal conductivity measurement.

Another important aspect of modeling the thermal conductivity is predicting its change under irradiation. Therefore, mesoscale thermal conductivity measurements are also needed for samples with varying amounts of irradiation damage. Such samples could be irradiated with ion-irradiation or using rabbit tests in a test reactor. The microstructures would need to be well characterized before or after the thermal conductivity measurement.

\subsubsection{Uncertainty Quantification}

While measurements have been conducted on the thermal conductivity of unirradiated $\mathrm{UO}_{2}$, these measurements were conducted on polycrystal samples with impurities, GBs, and some porosity. Thus, using these values for the single crystal thermal conductivity of pure $\mathrm{UO}_{2}$ introduces error into the calculation. In addition, no measured values are available for the GB thermal resistance or for the impact of defects on the thermal conductivity. These values have been calculated using MD simulations, but these values have significant uncertainty.

As with the grain growth and fission gas models, no uncertainty quantification has conducted on the effective thermal conductivity measurements in MARMOT.

DAKOTA could be effectively used to determine how the uncertainty of the various values for the local thermal conductivity impact the effective thermal conductivity.

\subsection{Fracture}

Fuel pellet fracture directly impacts fuel performance by reducing the gap between the fuel and cladding, reducing the stress within the material, creating more avenues for fission gas release, and decreasing the thermal conductivity. However, fracture is 
considered in traditional fuel performance codes either with a purely empirical relocation model or with a semi-empirical smeared crack model. As part of the NEAMS program, we are developing a mechanistic fracture model for BISON in which the crack behavior is a function of temperature, grain size, and GB bubble density. We are developing the macroscale model using atomistic simulations, as well as MARMOT simulations.

In MARMOT we have implemented a phase field fracture model that can model arbitrary crack propagation through a microstructure both in 2D and 3D. We model the cracking behavior in $\mathrm{UO}_{2}$ using fracture information for both intra- and intergranular fracture from MD simulations. We are using the model to quantify the impact of grain size and GB bubble density on fracture in $\mathrm{UO}_{2}$. The MARMOT results will be used to develop the engineering scale fracture model for BISON.

\subsubsection{Verification}

There are certain sample geometries for which the fracture behavior is known, and therefore can be used to verify the fracture behavior predicted by a model. Presently, mode I fracture in Single Edge Notch Tensile (SENT) specimen has been simulated and verified with [4.4.1]. The workability of the model to capture arbitrary crack path has also been verified by performing fracture simulation of a heterogeneous material under tensile loading. We are currently comparing the fracture behavior predicted by the phase field model to that predicted by MD simulations on an identical domain to further verify the fracture model in MARMOT.

\subsubsection{Validation}

The fracture behavior of $\mathrm{UO}_{2}$ depends on several factors including temperature, grain size, porosity, and stoichiometry. With increasing temperature, the fracture behavior may change from brittle to brittle with limited local plasticity to ductile. Regarding grain size, the fracture toughness increases with decreasing grain size, due to an increase in the amount of intergranular fracture [4.4.2]. For sintered $\mathrm{UO}_{2}$, the fracture toughness was found to increase with increasing porosity [4.4.3]. Validation data are desired regarding the effects of temperature, grain size and porosity.

Corresponding to the scales associated with the phase field model and MD, two sets of validation experiments are needed.

Indentation tests of sintered $\mathrm{UO}_{2}[4.4 .4,4.4 .5]$ are suggested to validate the phase field model regarding the impact of grain size. In these experiments, the samples need to be well characterized regarding their microstructure, including grain size, porosity and stoichiometry. The fracture toughness and the fracture morphology obtained from experiments will be compared with those predicted by phase field modeling using the same microstructure. Indentation experiments at different temperatures are also desired. The current phase field fracture model focuses on brittle fracture. By coupling with a crystal plasticity model in the future, it will be possible to simulate the transition in fracture behavior with increasing temperature. 
Currently there have been no nanoscale experiments investigating the fracture behavior of $\mathrm{UO}_{2}$. However, such information is highly desired to improve our understanding of the fundamental fracture mechanisms and to validate the results obtained by MD simulations [4.4.6]. Suggested experiments include nanoindentation within a high-resolution microscope so that the atomic scale information at crack tips can be extracted and compared with MD simulations. Tests on both single-crystal and well-designed bicrystal samples are suggested to explore the competition between inter- and intragranular fracture and possible GB anisotropy.

\subsubsection{Uncertainty Quantification}

Besides temperature, average grain size and porosity, the fracture behavior of $\mathrm{UO}_{2}$ can also be affected by other microstructure factors, which may not be accurately characterized experimentally, such as stoichiometry, pore size distribution, and impurity level. While comparing with modeling results, the uncertainties in these factors need be quantified, e.g., using DAKOTA or other approaches. On the other hand, the results produced by MD simulations are usually dependent on the choice of interatomic potential [4.4.7]. The corresponding uncertainties also need to be estimated.

\section{Path forward, Conclusions}

MARMOT is a critical part of the MBM suite of fuel performance codes being developed as part of the NEAMS Fuels Product Line. It is used to investigate mesoscale microstructure evolution and to develop mechanistic macroscale materials models based on microstructure rather than burnup. In order to establish confidence and to quantify the uncertainty in MARMOT calculations, a significant $\mathrm{V} \& \mathrm{~V}$ effort is needed. Most of the models within MARMOT have been verified against analytical models, though more verification is needed. In addition, more validation should be conducted in comparison with MD simulation results.

While some validation of MARMOT has been conducted against published experimental data, the majority of the data needed to validate MARMOT needs to be collected. The data required for the validation effort are described in this document, but we also summarize them in Table 5.1. These experiments are difficult to perform and require highly specialized capabilities, and therefore can only be conducted at certain Laboratory and University facilities. Possible avenues for funding researchers to obtain these data include the NEUP program, the FCRD program, and NEAMS-funded experiments. 
Table 5.1: Summary of experiments needed for MARMOT validation

\section{$\mathrm{GB}$ migration and grain growth in $\mathrm{UO}_{2}$}

- Bicrystal experiments on different GB types

- Polycrystal annealing experiments with well characterized initial microstructure

- Polycrystal annealing experiments with various pore densities and wellcharacterized initial microstructure

\section{Fission gas migration in $\mathrm{U}_{2}$}

- Experiments on unirradiated samples with various concentrations of Xe

- Experiments on ion-irradiated samples using Xe ions

- Low exposure reactor experiments with well characterized microstructure before and after irradiation

\section{Thermal conductivity in $\mathrm{UO}_{2}$}

- Single crystal thermal conductivity measurements in stoichiometric and offstoichiometric $\mathrm{UO}_{2}$.

- GB thermal resistance measurements using bicrystal or large grained samples

- Measurement of the impact of dispersed Xe on thermal conductivity

- Measurements of the thermal conductivity across well-characterized polycrystalline samples

- Measurement of thermal conductivity across well-characterized polycrystalline samples with increasing amounts of irradiation damage

\section{Fracture behavior in $\mathrm{UO}_{2}$}

- Micro-indentation tests of well-characterized sintered $\mathrm{UO}_{2}$ samples with various grain sizes, porosity, and stoichiometry

- Nano-indentation within a high-resolution microscope on single-crystal and well-designed bicrystal samples

The NEUP program has included validation experiments for MARMOT in its call for the last two years. However, as the MARMOT development team has no control over the selection of NEUP projects and no required interaction with the projects once they are funded, it is proving difficult to obtain the correct data from these projects. In addition, the NEUP program does not have enough funding to obtain all of the data required for the MARMOT validation.

The FCRD program has worked with NEAMS and specifically with the MARMOT development team in the past to develop experiments that will provide needed data to validate the MARMOT models laid out in this document. Some of this work has been started, but due to a change in focus of the FCRD program away from fundamental $\mathrm{UO}_{2}$ studies, these experiments have been canceled or severely delayed. 
This represents an unfortunate disconnect between the FCRD and NEAMS programs and should be remedied, if possible.

Some experimental work could be directly funded by the NEAMS program, as this would provide the most control and the best interaction between the experimental and modeling teams. This approach is currently being used to fund experimental work on measuring the single crystal $\mathrm{UO}_{2}$ thermal conductivity for stoichiometric and off-stoichiometric samples. This approach is not feasible for all of the necessary data unless the NEAMS Fuels Product Line funding is significantly increased.

The optimal approach to collect the data required for the MARMOT validation is probably a combination of the three approaches mentioned above. Continued funding of MARMOT validation through the NEUP program could be a useful means to obtain some data, and direct involvement of the MARMOT development team in the projects would help to ensure that the experiments are relevant. If the FCRD program returns to funding some fundamental $\mathrm{UO}_{2}$ projects, they could obtain another portion of the required data. Finally, the NEAMS program could fund experiments to provide critical data that is not being provided by NEUP projects or by the FCRD program.

\section{References and Additional Material}

[3.1.1] MR Tonks, DR Gaston, PC Millett, D Andrs, and P Talbot, Comp. Mat. Sci., 51 (2012) 20-29.

[3.1.2] Multiphysics Object Oriented Simulation Environment, GitHub repository, https://github.com/idaholab/moose.

[3.1.3] L.Q. Chen. Annu. Rev. Mater. Res., 32 (2002) 113-40.

[3.1.4] MR Tonks, PC Millett, P Nerikar, S Du, D Andersson, CR Stanek, D Gaston, D Andrs, and R Williamson, J. Nucl. Mater. 440 (2013) 193-200.

[4.1.1] MR Tonks, Y Zhang, SB Biner, PC Millett, and X Bai, Acta Mater., 61 (2013) 1373-1382.

[4.1.2] MR Tonks, Y Zhang, X Bai and PC Millett, Mater. Research Letters 2 (2013), 23-28.

[4.1.3] N Moelans, B Blanpain, and P Wollants, Phys Rev B 78 (2008) 024113.

[4.1.4] G Gottstein, LS Shvindlerman, Grain boundary migration in metals: thermodynamics, kinetics, applications. CRC Press, Boca Raton; 1999.

[4.1.5] J Ainscough, B Oldfield, and J Ware. J. Nucl Mater 49:2 (1973) 117-128.

[4.1.6] PV Nerikar, K Rudman, TG Desai, D Byler, C Unal, and KJ McClellan, J. Amer. Ceram. Soc. 94:6 (2011) 1893-1900.

[4.2.1] PC Millett and MR Tonks, Current Opinion Solid State Mater. Sci., (2010) doi:10.1016/ j.cossms.2010.10.002.

[4.2.2] MR Tonks, SB Biner, PC Millett, and DA Andersson, Int. Conf. Math. Comp. Methods Applied to Nucl. Sci. \& Eng. (M\&C2013), Sun Valley, ID, May 2013.

[4.2.3] PC Millett, MR Tonks and SB Biner, J. Applied Physics, 111:8 (2012) 083511083511-7. 
[4.2.4] IO Usov, RM Dickerson, PO Dickerson, ME Hawley, DD Byler, and KJ McClellan, J. Nucl. Mater., 437 (2013) 1-5.

[4.3.1] PC Millett and MR Tonks, J. Nucl. Mat., 412:3 (2011) 281-86.

[4.3.2] PC Millett, MR Tonks, K Chockalingam, Y Zhang, and SB Biner, J. Nucl. Mater. 439 (2013) 117-122.

[4.3.3] MR Tonks, PC Millett, P Nerikar, S Du, D Andersson, CR Stanek, D Gaston, D Andrs, and R Williamson, J. Nucl. Mater. 440 (2013) 193-200.

[4.4.1] C Miehe, F Welschinger, and M Hofacker, Int. J. Num. Methods. Eng. 83 (2010) 1273-1311.

[4.4.2] AG Evans and RW Davidge, J. Nucl. Mater. 33 (1969) 249.

[4.4.3] TRG Kutty, KN Chandrasekharan, JP Panakkal, and JK Ghosh, J Mater. Sci. Lett. 6 (1987) 260.

[4.4.4] HJ Matzke and T Inoue, J. Nucl. Mater. 91, (1980) 205.

[4.4.5] K. Kapoor, Ansar Ahmad, A. Laksminarayana, G.V.S. Hemanth Rao, J. Nucl. Mater. 366, (2007) 87.

[4.4.7] Y. F. Zhang, X. Y. Liu, P. C. Millett, M. Tonks, D. A. Andersson, and B. Biner, J. Nucl. Mater. 430 (2012) 96.

[4.4.7] K. Govers, S. Lemehov, M. Hou, M. Verwerft, J. Nucl. Mater. 366 (2007) 161. 